\title{
Unser langjähriger Zusammenarbeitspartner, die WIROMA AG, bietet DR-Systeme* für die Arztpraxis
}

\section{Das Speicherfoliensystem für die Arztpraxis mit Akquisitions- und Befundungsstation in einem}

- Auslesegerät Proscan 35

- PC mit Touchscreen

- Akquisitionssoftware (inkl. Modul zur Anbindung an ein Patientenverwaltungsprogramm)

- 4 Speicherfolien mit Kassetten $(18 \times 24 \mathrm{~cm}, 24 \times 30 \mathrm{~cm}, 35 \times 43 \mathrm{~cm}, 18 \times 35 \mathrm{~cm})$

- Befundungsmonitor (z. B. ElZO GS220) mit Grafikkarte

- Diagnosesoftware (inkl. Modul zum Brennen einer Patienten-CD)

- Backup-Lösung (Hard- und Software) inbegriffen

- Installation und Abnahmeprüfungen inbegriffen

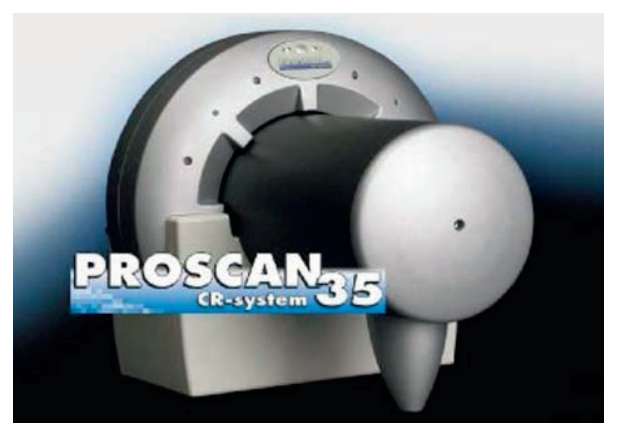

Zum unschlagbaren Preis von

Fr. 39900.- (exkl. MWSt.)

* DR-System = Direct-Radiography-System (Flat Panel).

Gerne beraten wir Sie kostenlos und unverbindlich

\section{Antworttalon}

Ich/wir sind interessiert an:

$\square$ Beratung/Planung

$\square$ Film/Chemie

$\square$ Digitales Röntgen

Name:

Adresse:

Tel.

$\square$ Röntgenanlagen

$\square$ Strahlenschutz

$\square$ Personendosimetrie

Vorname:

PLZ / Ort: $\square$ Zubehör

$\square$ Qualitätssicherung

Beste Anrufzeit: 